\title{
Consumo de drogas de síntesis en estudiantes de secundaria del Principado de Asturias (España)
}

\author{
Fernández ludeña, J.(1); Álvarez Fresno, e. (1); Secades Villa, R. ${ }^{(2)}$; Jiménez García, J.M. ${ }^{(1)}$; \\ Cañada Martínez, A. ${ }^{(3)}$; Donate Suárez, I. ${ }^{(3)}$; Fernández Hermida, J.R..$^{(2)}$; Vallejo Seco, G. ${ }^{(2)}$ \\ (1) Colegio Oficial de Psicólogos del Principado de Asturias \\ (2) Departamento de Psicología. Universidad de Oviedo. \\ (3) Consejería de Salud y Servicios Sanitarios del Principado de Asturias.
}

\section{RESUMEN}

El objetivo de este estudio es analizar la situación del consumo de drogas de síntesis entre la población juvenil del Principado de Asturias, así como identificar las variables relacionadas con el uso de estas sustancias. La muestra se compuso de 1597 estudiantes de educación secundaria de esta Comunidad. Los resultados señalan que el $8 \%$ de los jóvenes encuestados había consumido drogas de diseño alguna vez, el 7\% había consumido a lo largo del último año y el 4,7\% en los últimos 30 días. La prevalencia aumenta con la edad y los hombres consumen más que las mujeres. Las variables relacionadas con el consumo son, entre otras, pobre rendimiento académico, actitudes tolerantes hacia las drogas, vivir solo con uno de los padres, tener mala relación con la familia y consumo de drogas de los amigos y la familia. Estos resultados indican un crecimiento importante del consumo de estas sustancias en Asturias y apuntan la necesidad de la puesta en marcha de estrategias que frenen este incremento entre la población juvenil.

Palabras clave: Drogas de síntesis, éxtasis, jóvenes, factores de riesgo.

\section{ABSTRACT}

The objective of this study is to analyze the situation of designer drug use in young people in Asturias (northern Spain), and to identify the variables related to the consumption of these substances. The sample was made up of 1597 students from secondary schools in this Autonomous Region. Results show that $8 \%$ of the young people interviewed had consumed designer drugs at some time, $7 \%$ had taken them during the previous year and $4.7 \%$ had used them during the previous 30 days. Prevalence of use increased with age, and males consumed more than females. Variables related to consumption are, among others, poor academic performance, tolerant attitudes towards drugs, living with only one parent, strained relations with family, and drug use by friends and family members. These results point to considerable growth in consumption of these substances among the adolescent population in Asturias, and suggest the need for strategies to halt this increase.

Key words: Designer drugs, ecstasy, adolescents, risk factors.

\section{INTRODUCCIÓN}

L a extensión del uso de drogas de síntesis por la geografía española, especialmente MDA y sus congéneres (MDMA, MDEA y MBDB), desde finales de los años ochenta y comienzos de los noventa, ha supuesto un cambio en las pautas de ocio de fin de semana de un amplio sector de la juventud con respecto a generaciones anteriores.

Sin embargo, existen pocos trabajos españoles publicados sobre drogas de diseño en comparación con la producción global en drogodependencias, y menos aún, estudios experimentales y epidemiológicos (Aleixandre, Valderrama y Cervera, 2000).

Como estudios pioneros destacan los realizados por el equipo de Gamella (Gamella, Álvarez Roldán y Meneses, 1996; Gamella y Álvarez Roldán, 1997) en cinco comunidades Autónomas de España, por de la

Este estudio ha sido financiado por la Consejería de Salud y Servicios Sanitarios (Plan sobre Drogas) del Principado de Asturias. 
Fuente et al. (1997) y por Calafat, Sureda y Palmer (1997) en Palma de Mallorca.

El instituto IREFREA (2001), a través del equipo de Amador Calafat, llevó a cabo en 1998 una investigación en nueve ciudades europeas sobre el policonsumo en ambientes recreativos. El éxtasis era consumido por el $37,5 \%$ de los jóvenes alguna vez en su vida, algo menos que la muestra Europea. Las pautas de consumo eran más bien esporádicas, aunque un $10 \%$ lo consumía de forma semanal y mensual. El estudio demostró que el policonsumo se presenta como un hecho indiscutible entre los jóvenes durante los fines de semana. El alcohol se mezcla con casi todas las sustancias, así como el cannabis y la cocaína.

La Encuesta sobre Drogas a la Población Escolar realizada por el Plan Nacional sobre Drogas en el año 1998 (1999) mostraba que el consumo de psicoestimulantes reflejaba cambios notables con respecto a la situación existente en 1996. El consumo de éxtasis, que había crecido entre los escolares de forma espectacular en el período 1994-96, se había reducido en 1998 hasta situarse por debajo de los niveles que tenía en 1994. De hecho, los porcentajes de consumidores se habían reducido en todas las edades, tanto en varones como en mujeres.

Los últimos datos de que disponemos corresponden a la encuesta que el Plan Nacional sobre Drogas realizó a la población escolar en el año 2000 (2001). En ella se observa un incremento significativo en el consumo de drogas de diseño. Así, respecto al consumo alguna vez de éxtasis u otras drogas de diseño, contestan afirmativamente un $5,70 \%$ de la muestra, lo que contrasta con el 3,5\% de la encuesta de 1998 . Respecto al consumo en los últimos 12 meses, el porcentaje de la encuesta del 2000 alcanza al 4,60\%, siendo en la de 1998 de un 2,5\%.

En lo que se refiere a la situación del uso de estas drogas en Asturias, la Encuesta de Población Escolar revela que la evolución del consumo de éxtasis (consumo alguna vez) pasó desde un 3\% en el año 1994, hasta el 3,9\% en el año 96 , y de un $2,5 \%$ en el año 1998, al 4,80\% en el año 2000.

El estudio realizado por Sáiz Martínez et al. (2001) con estudiantes de secundaria de Oviedo (Asturias) encontró unas prevalencias vida, último año y último mes, de consumo de MDMA de 3,8\%, 2,7\% y $1,6 \%$ respectivamente.

A estos datos, se añaden diferentes informaciones de fuentes periodísticas y judiciales que parecen apuntar un uso creciente de estas sustancias entre la población joven del Principado de Asturias.

Es de gran interés, por tanto, conocer con mayor detalle cómo evoluciona la incidencia del consumo de estas sustancias en la población joven del Principado y analizar diversos aspectos relacionados con el uso de éstas: los patrones de consumo, las motivaciones para su uso, los efectos que buscan los jóvenes, la percepción de los riesgos y daños que pueden causar dichos consumos, la información que tienen los jóvenes escolares sobre las sustancias, así como la actitud hacia éstas y su consumo.

Dicho conocimiento nos permitiría planificar y diseñar pautas de actuación, con el fin de prevenir el consumo y, en la medida de lo posible, reducir los riesgos asociados al uso de tales sustancias en la población juvenil asturiana.

En concreto, los objetivos de este estudio son los siguientes: 1) determinar la prevalencia del consumo de drogas de síntesis y otras drogas en los jóvenes escolarizados entre 14 y 18 años del Principado de Asturias, 2) conocer las características y los patrones de uso de estas sustancias, 3) conocer el nivel de información sobre las drogas de síntesis que tienen los escolares, 4) conocer las motivaciones y los efectos del consumo de estas sustancias y 5) identificar las variables relacionadas con el uso de éxtasis en esta población.

\section{MATERIAL Y MÉTODOS}

\section{Muestra}

Para la selección de los participantes se utilizó un muestreo por conglomerados polietápico, con los conglomerados seleccionados con probabilidad proporcional a su tamaño. Los conglomerados que se establecieron fueron las Áreas Sanitarias del Principado de Asturias (I-II, III, IV, V, VI, VII y VIII) y el tipo de colegio (publico y privado-concertado).

La unidad muestral fue el aula escolar. En cada centro escolar se seleccionaron dos aulas al azar, siendo encuestados todos sus alumnos.

La muestra se seleccionó de entre una población total de 40.033 escolares de toda la Comunidad Autónoma del Principado de Asturias, nacida entre los años 1982 y 1986, distribuida en 120 centros de educación secundaria. La muestra final seleccionada se compuso de 1597 sujetos $(54,7 \%$ mujeres y $45,3 \%$ hombres). La edad media es de16 años (rango: 14-18 años, y $\sigma=1,29$ ).

El $65,5 \%$ de los participantes pertenecía a 22 centros públicos y el $34,5 \%$ a 14 colegios privados y privado-concertados. El $50,5 \%$ vivía en alguna de las tres grandes ciudades de Asturias (Oviedo, Gijón y Avilés), el $28,6 \%$ en capitales de concejo y el $19,4 \%$ en localidades de menor tamaño.

El 23\% eran hijos únicos y sólo el $11 \%$ tenían 3 ó más hermanos. El 25,4\% había repetido curso escolar alguna vez y el 6,8\% había repetido 2 ó más cursos. 


\section{Variables e Instrumentos}

El instrumento de recogida de información fue un cuestionario elaborado "ad hoc" para la investigación. Se componía de 47 ítems, algunos de ellos extraídos de la Encuesta de Población Escolar del Plan Nacional sobre Drogas.

Las preguntas que recogía el cuestionario hacían referencia a los siguientes factores: (1) datos sociodemográficos, (2) datos escolares, (3) información sobre drogas de síntesis, (4) actitud hacia las drogas de síntesis, (5) opinión acerca de las drogas de síntesis, (6) consumo de drogas de síntesis: patrones, tipos, contextos, efectos, motivaciones, (7) consumo de otras drogas, (8) pautas de ocio y ocupación del tiempo, (9) relación familiar y (10) consumo de drogas en el ámbito familiar y de amigos.

\section{Procedimiento}

En los meses de marzo y abril de 2001 se contactó telefónicamente con los centros elegidos previamente al azar. Los cuestionarios, que eran anónimos, se aplicaron de forma colectiva en horario escolar.

Los aplicadores fueron tres psicólogos, miembros de la Comisión de Conductas Adictivas del Colegio Oficial de Psicólogos de Asturias.

\section{Análisis de datos}

Se llevaron a cabo diferentes análisis de frecuencias para describir las características de la muestra y las variables relacionadas con el consumo de drogas de diseño. Se realizaron análisis bivariados (chi-cuadrado y t de Student) para comprobar la relación entre el consumo de drogas de diseño y otras variables medidas con el cuestionario. El nivel de confianza fue del 95\%.

\section{RESULTADOS}

\section{Consumo de drogas de diseño}

El 8\% de los jóvenes encuestados habían consumido drogas de diseño alguna vez, el 7\% había consumido a lo largo del último año y el 4,7\% en los últimos 30 días.

De los 86 jóvenes que habían consumido en el último mes, el 2,1\% lo habían hecho de 1 a 2 días, el 1,9\% de 3 a 5 días y sólo el 0,7\% 6 días o más.

La edad media de los consumidores es de 16,35 y la de los no consumidores de 15,73. La tabla 1 nos muestra el consumo de éxtasis en función de la edad.

\begin{tabular}{|lcccccc|}
\hline \multicolumn{5}{|c|}{$\begin{array}{c}\text { Tabla 1. Porcentaje de consumidores } \\
\text { en función de la edad }\end{array}$} \\
\hline Edad & 14 años & 15 años & 16 años & 17 años & 18 años \\
\hline$\%$ Consumidores & 1,9 & 5,2 & 10,5 & 8,0 & 12,15 \\
\hline
\end{tabular}

En cuanto al género, hay un $8,6 \%$ de hombres consumidores frente al 5,3\% de mujeres.

En la Tabla 2 se resumen los datos relativos a la adquisición de las pastillas (para la lectura de la tabla se debe tener en cuenta que el cuestionario permitía más de una respuesta).

Tabla 2. Forma de adquisición de las pastillas (\%)

\section{Fuente de obtención del dinero}

$\begin{array}{lr}\text { Paga familiar } & 76,2 \\ \text { Trapicheos } & 30,5 \\ \text { Trabajo } & 17,4 \\ \text { Pequeños robos } & 8,4 \\ \text { Otros } & 12,7\end{array}$

\begin{tabular}{|c|c|}
\hline \multicolumn{2}{|l|}{ Lugar de adquisición } \\
\hline Calle & 59,3 \\
\hline Discotecas & 55,6 \\
\hline Bares & 32,8 \\
\hline Centro escolar & 5 \\
\hline Otros & 19,7 \\
\hline \multicolumn{2}{|c|}{ Personas que las suministran } \\
\hline Amigos & 76,3 \\
\hline Pequeños vendedores & 45,7 \\
\hline Novio/a & 14,3 \\
\hline Otras fuentes & 6,2 \\
\hline
\end{tabular}

Los datos que reflejan el contexto de consumo se muestran en la Tabla 3.

\section{Tabla 3. Contexto de consumo de las drogas de diseño (\%)}

\section{Momento de consumo}

Fin de semana

Sólo en ocasiones especiales

Cualquier día de la semana

Otras ocasiones

\section{Lugar de consumo}

Discotecas

Bares o afters

Naves, carpas o alrededores

En la calle

En mi casa o en casa de amigos

Otros lugares

\section{Personas con las que consume}

Preferentemente con amigos

A veces solo y a veces con amigos

Preferentemente solo

Con mi novio/a

Con otros

La media de pastillas consumidas a la semana es de 2,39 $(\sigma=2,19)$. El $40,7 \%$ de los consumidores 
habituales, consume sólo una pastilla a la semana. El $29,6 \%$ dos y el $29,7 \%$ consume tres o más pastillas (es decir, el 1,1\% de la población general). Un 6,3\% de los consumidores habituales consumen 5 pastillas y un 1,8\% (el 0,1\% de la población total) consumen 6 , el mismo porcentaje sería para cualquier cantidad superior.

El número promedio de pastillas por sesión de consumo (tiempo bajo el que se está bajo los efectos de las pastillas de forma continuada) es de 2 ( $\sigma=$ $1,22)$. Casi la totalidad, el $89,7 \%$ no consume más de tres pastillas. El 40,4\% y el 41,3\% afirma tomar 1 ó 2 respectivamente. El 19,4\% consume tres o más pastillas por sesión. Los que consumen 4 ó 5 pastillas son el $5 \%$ y el 3,5\% respectivamente.

La media de dinero gastado en pastillas a la semana es de $18 €$, siendo el $23,5 \%$ y el $31,9 \%$ los que gastan 6 y $12 €$ respectivamente.

En cuanto a los motivos para consumir este tipo de drogas, cerca de la mitad afirman consumirlas para divertirse $(47,8 \%)$ o experimentar nuevas sensaciones (44,3\%). Le siguen el bailar más tiempo o disfrutar más del baile $(42,7 \%)$ y escapar de la realidad (35,3\%). Sólo el 10,1\% dice utilizarlas para relacionarse mejor y el 6,4\% para mantener relaciones sexuales. Hay un 14,1\% que no manifiesta ninguna razón en particular.

La mayor parte de los jóvenes consumidores de drogas de síntesis son policonsumidores, es decir, consumen también otro tipo de drogas. El 68,7\% de la muestra declara consumir drogas de síntesis acompañadas de alcohol y el 60,5\% de cannabis. El porcentaje de los que lo acompañan de cocaína desciende al 26,2\%, el $13 \%$ de alucinógenos, y son ya muy pocos (alrededor del $5 \%$ ) los que usan drogas de síntesis junto con anfetaminas o tranquilizantes y un $1,5 \%$ con opiáceos.

Los efectos inmediatos experimentados después de haber consumido drogas de diseño son, por este orden: euforia y energía, y sensación de felicidad. Casi tres de cada cuatro jóvenes afirman experimentar agotamiento 24 horas después de consumir y casi la mitad apatía, dificultad para concentrarse, pérdida de apetito e incapacidad para estudiar.

\section{Consumo de Éxtasis y relación con otras variables}

A continuación se exponen los resultados que hacen referencia a la relación entre la variable consumo de drogas de diseño en el último año y algunas otras variables medidas a través del cuestionario. Sólo se incluyen aquellas variables que han mostrado tener una relación estadísticamente significativa con la variable dependiente.

En la Tabla 4 se resumen los datos extraídos de estos análisis bivariados.
La edad media de los consumidores es de 16,35 y la de los no consumidores 15,73; es decir, el consumo aumenta con la edad.

Los hombres consumen más que las mujeres, $8,6 \%$ frente al 5,35\% .

Los adolescentes que más consumen afirman tener más información acerca de las drogas de síntesis que los no consumidores.

Los consumidores tienen unas actitudes más tolerantes hacia estas sustancias (media de 17,44) con relación a los no consumidores (media de 14,02).

Los jóvenes que viven sólo con uno de sus padres tienen mayor probabilidad de consumir drogas de síntesis $(9,3 \%)$ que aquellos que viven con ambos padres $(6,7 \%)$ y menos que los que no viven con ninguno de sus progenitores $(18,7 \%)$.

Los consumidores tienen peor rendimiento académico (medido a través de la nota media del curso anterior y de los cursos repetidos) que los no consumidores. Así entre los suspensos se encuentran un $16,9 \%$ de consumidores, mientras que estos bajan al $9,8 \%$ en los aprobados y así sucesivamente hasta el $4,1 \%$ en los notables y ninguno entre los sobresalientes. Entre los repetidores de curso hay un 15,5\% de consumidores, frente al 4,2\% entre los que no habían repetido ningún curso.

En cuanto al consumo por Áreas Sanitarias en las que se divide la Comunidad Autónoma Asturiana, se observa mayor consumo en el Area de Avilés (14\%) y Oviedo $(9,1 \%)$. Las siguen las de Occidente $(6 \%)$, Gijón (5,9\%), Oriente (5\%), Caudal $(2,7 \%)$ y, por último, Nalón (1\%).

El consumo se concentra, preferentemente, en las ciudades de mayor tamaño (Oviedo, Gijón y Avilés), con el 9\% como promedio, disminuye al 5,3\% en el resto de capitales de concejo y al 3,9\% en los ámbitos rurales.

Los jóvenes que han consumido drogas de diseño dicen tener peores relaciones familiares (media de1,74) en comparación a los no consumidores (media de 1,50).

En general, los amigos y la familia de los consumidores consumen a su vez más drogas, legales e ilegales, que los amigos y la familia de los no consumidores.

\section{DISCUSIÓNY CONCLUSIONES}

El objetivo de este estudio era analizar la situación del consumo de drogas de diseño entre los jóvenes asturianos.

Los datos aportados pueden ser de gran utilidad de cara a planificar y diseñar pautas de actuación con el fin de prevenir el consumo de estas sustancias y, 
Tabla 4. Relación entre el consumo de drogas de diseño y otras variables

\begin{tabular}{|lrr|}
\hline Variable & $\left(\chi^{2} / \mathbf{t}\right)$ & $(\mathbf{p}<)$ \\
\hline Edad & 29,513 & 0.000 \\
Género & 6,546 & 0.008 \\
Personas con las que convive & 8,861 & 0.021 \\
Nota media del curso anterior & 40,671 & 0.000 \\
Cursos repetidos & 56,466 & 0.000 \\
Área Sanitaria & 31,964 & 0.000 \\
Tipo de población en la que vive & 11,629 & 0.002 \\
Información sobre drogas de diseño & 26,107 & 0.000 \\
Actitudes hacia las drogas de diseño & $-9,002$ & 0.000 \\
Relación con la familia & $-5,399$ & 0.000 \\
Consumo de drogas en la familia & $-4,954$ & 0.000 \\
Consumo de drogas en los amigos & 13,245 & 0.000 \\
\hline
\end{tabular}

en la medida de lo posible, reducir los riesgos asociados a su uso en la población juvenil asturiana.

Los resultados obtenidos muestran que el consumo de drogas de síntesis entre la población escolar creció de forma espectacular en Asturias en el periodo de 1998-2001, con respecto a la media nacional encontrada por el Plan Nacional sobre Drogas. De hecho, el porcentaje encontrado en nuestro estudio duplica las cifras de la encuesta nacional. El éxtasis tiene actualmente unos niveles de prevalencia sensiblemente inferiores al del alcohol o del cannabis, pero superiores a la cocaína, anfetaminas y alucinógenos.

La alta prevalencia de consumo de drogas de síntesis encontrada puede resultar bastante llamativa. No obstante, no encontramos ninguna razón para pensar que los resultados de nuestro estudio estén inflados con respecto a la Encuesta Escolar Nacional, entre otros motivos, porque los procedimientos para la recogida de datos utilizados en ambos casos fueron similares. Por otra parte, determinadas informaciones periodísticas y policiales de los últimos meses parecen avalar también el dato del incremento del consumo de estas sustancias en esta Comunidad Autónoma.

Es importante señalar también que el porcentaje de consumidores encontrados en este estudio se asemeja bastante al hallado en un trabajo similar en los adolescentes del municipio de Oviedo (Secades Villa y Fernández Hermida, 2001).

Cabe preguntarse si este alto porcentaje de adolescentes que afirman haber probado las drogas de síntesis, responde a un fenómeno pasajero y circunstancial, motivado por determinadas causas que desconocemos (por ejemplo, un incremento puntual de la oferta), o se trata más bien de un cambio relativamente estable en los patrones de uso de estas sustancias. Obviamente, se necesitarían nuevos estudios para poder aclarar esta cuestión.

Como en otras investigaciones, los resultados muestran que el consumo de éxtasis es significativa- mente superior entre los hombres, que la edad media de los consumidores es superior a los no consumidores y que el consumo aumenta con la edad. Junto a esto, es importante resaltar el hecho de que el porcentaje de consumidores jóvenes (14 años) es también sensiblemente superior al hallado en la Encuesta Escolar del Plan Nacional sobre Drogas.

Se consume más en los grandes núcleos de población del centro de la provincia que en las poblaciones más pequeñas. Destacan, sobre todo, las cifras de Avilés y Oviedo, mientras que el consumo es significativamente inferior en los núcleos más reducidos de las cuencas mineras y el occidente y oriente asturianos.

Por otra parte, no existen diferencias significativas entre los centros públicos y privados, salvo en el hecho de que los alumnos de los centros privados dicen poseer más información que los de los públicos.

En cuanto al patrón habitual de consumo, los datos muestran que el número medio de pastillas consumidas por sesión es inferior a los datos obtenidos a nivel nacional. También lo es el número de personas que han consumido tres ó más pastillas en una sola sesión.

Por otra parte, los datos de este estudio vuelven a confirmar, en contra de una creencia bastante extendida, que la combinación de éxtasis con otras sustancias es muy frecuente, especialmente con el alcohol y el hachís.

Si a estos datos unimos el hecho de que algo más de un tercio de los escolares que consumieron en el último año no ha consumido más de una pastilla a la semana, podemos pensar que ha aumentado el número de personas experimentadoras de estas drogas, pero no la intensidad de su uso.

Para algo más de la mitad, el momento fundamental de consumo es el fin de semana y los lugares más frecuentes son las discotecas, seguido de los bares o pubs.

Los motivos para consumir son similares a los señalados en la Encuesta de la Población Escolar del año 1998, así como en otras investigaciones. Estos motivos son, para cerca de la mitad de los encuestados, divertirse y experimentar sensaciones, y para algo menos de la mitad, bailar más tiempo o disfrutar más del baile.

Ambos datos confirman la asociación del consumo de éxtasis con los contextos y las motivaciones de tipo lúdico, dato señalado reiteradamente por varios estudios similares publicados hasta la fecha.

El dinero para la compra de las pastillas proviene, fundamentalmente, de la paga familiar, y los proveedores más frecuentes son los amigos, siendo los lugares más habituales de adquisición, la calle y la discoteca, seguido de los bares.

Los efectos inmediatos experimentados después de haber consumido drogas de diseño coinciden fun- 
damentalmente con los ya aparecidos en estudios anteriormente publicados (Gamella y Roldán, 1997). Así casi la totalidad hablan de euforia y energía y ya con menor frecuencia la sensación de felicidad, atreverse a hacer cosas y experimentar más sensaciones.

Los efectos experimentados 24 horas después del consumo son el agotamiento, la apatía y la dificultad para concentrarse. No obstante, se debe decir que estos estados son difícilmente atribuibles sólo al efecto de las pastillas, ya que también pueden ser el resultado del tipo de actividad desarrollada intensa e ininterrumpida que suele acompañar al uso de estas sustancias.

Al menos, un tercio de los escolares consumidores de éxtasis ha experimentado diferentes problemas, como peleas o agresiones físicas, ausencias del colegio o pérdida de memoria, que atribuyen al consumo de dichas sustancias.

Tres de cada cuatro escolares afirman haber tenido algún tipo de información sobre las drogas de síntesis, considerando algo más de la mitad esa información muy útil o bastante útil. Un alto porcentaje se considera informado sobre los efectos perjudiciales y los lugares de consumo, pero muy pocos sobre el contenido de las pastillas. De hecho sólo la mitad de los consumidores afirman estar informados sobre dicho contenido.

En cuanto a las opiniones y actitudes acerca de estas sustancias, la mayoría piensa que las drogas de diseño enganchan y que pueden causar efectos perjudiciales, pero también piensan que la gravedad de esos efectos depende de que dicho consumo sea esporádico o habitual. Para la mitad de la población, conseguir drogas de síntesis les resultaría difícil o prácticamente imposible y un número algo superior afirman que nunca le han ofrecido dichas drogas.

Un dato de interés es que los consumidores dicen tener más cantidad de información que los no consumidores. Sin embargo, aquellos que han obtenido la información preferentemente de los profesores y de los medios de comunicación consumen menos que los que la tienen a través de otras fuentes, como son los amigos u otras personas que han consumido. De todos modos, son muy pocos (el 17\%) lo que dicen recibir información de los profesores.

Por lo que se refiere a la relación entre el uso de drogas de síntesis con otras variables valoradas en el estudio, se puede decir que, en general, el rendimiento escolar se encuentra muy asociado al consumo de pastillas, ya que existe un mayor porcentaje de consumidores entre los repetidores de curso y entre los que obtienen peores calificaciones.

Además, los que consumen tienen una actitud $u$ opinión más tolerante hacia este tipo de sustancias: no crea adicción, no son perjudiciales tomadas esporádicamente, se puede divertir uno más.

Las relaciones familiares y el uso de drogas en el grupo de amigos y en la familia también se han encon- trado altamente relacionadas con el consumo de estas sustancias entre los adolescentes. Así, una buena relación familiar parece ejercer como factor protector ante el consumo de drogas de síntesis. Lo contrario ocurre en aquellas familias en las que hay consumo de algún tipo de drogas ilegales (este hecho actúa como un factor de riesgo).

Los jóvenes que no viven con sus padres tienen una mayor probabilidad de consumir drogas de síntesis. El $13 \%$ de los que no viven con el padre consumen frente al $7 \%$ de los que sí viven.

Por último, aquellos que se relacionan con pandillas donde nadie o casi nadie fuma o consume alcohol, o donde nadie consume hachís, apenas consumen drogas de síntesis. Hay más probabilidad de consumir pastillas en los casos en que todos los amigos o la mayoría consume algún tipo de drogas, tanto legales como ilegales.

Como se puede apreciar, las variables relacionadas con el consumo de drogas de síntesis (factores de riesgo) en la población estudiada son muy similares a las encontradas en otros trabajos. Así, el pobre rendimiento académico, las actitudes permisivas hacia las drogas, las malas relaciones familiares y el consumo de drogas en el entorno (familia y grupo de amigos) parecen ser factores de riesgo para el consumo de este tipo de sustancias.

En conclusión, los resultados de este estudio debieran ser un toque de atención para los responsables de las instituciones públicas, ya que señalan la existencia de un problema creciente de consumo de drogas de síntesis en la población juvenil del Principado de Asturias, concentrado, sobre todo, en los núcleos de población urbana. De confirmarse esta tendencia, sería una prioridad intensificar los esfuerzos preventivos orientados específicamente a este tipo de sustancias. Entre otros contenidos, sería fundamental diseñar y aplicar estrategias con el fin de incrementar la percepción de riesgo de consumo de estas sustancias entre la población de riesgo.

\section{REFERENCIAS BIBLIOGRÁFICAS}

Aleixandre Benavent, R., Valderrama Zurián, J.C. y Cervera, G. (2000). Producción científica nacional e internacional en drogas de diseño (1988-1997). Adicciones, 12, 2, 195-205.

Calafat, A.; Sureda, M.P. y Palmer, A. (1997). Características del consumo de éxtasis en una muestra de universitarios y usuarios de discoteca. Adicciones, 9, 4, 529-555.

de la Fuente de Hoz, L., Rodríguez Arenas, M.A., Vicente Orta, J., Sánchez Payá, J. y Barrio Anta, G. (1997). Epidemiología del consumo de drogas de diseño en España. Medicina Clínica, 108, 54-61.

Gamella, J.F., Álvarez Roldán, A y Meneses, C. (1996). Drogas de síntesis en España: datos para una investigación 
antropológica. XXIII Jornadas Nacionales de Socidrogalcohol. Libro de actas (117-126), Oviedo.

Gamella, J.F. y Álvarez Roldán, A. (1997). Drogas de Síntesis en España. Patrones de adquisición y consumo. Un estudio antropológico en cinco Comunidades Autónomas. Madrid: Delegación del Gobierno para el Plan Nacional sobre Drogas.

IREFREA (2001). Salir de marcha y consumo de drogas. Disponible en: http:// www.mir.es/pnd/doc/observat/estudios/irefrea/ 1 Febrero 2001.

Plan Nacional sobre Drogas (1999). Encuesta sobre Drogas a la población escolar 1998. Madrid: Delegación del Gobierno para el Plan Nacional sobre Drogas.
Plan Nacional sobre Drogas (2001). Encuesta Domiciliaria sobre uso de drogas 2000. Madrid: Delegación del Gobierno para el Plan Nacional sobre Drogas.

Sáiz Martínez, P.A., González García-Portilla, M.P., Paredes Ojanguren, B., Delgado González, J.M., López Rodríguez, J.L., Martínez Barrondo, S. y Bobes García, J. (2001). Consumo de MDMA (éxtasis) en estudiantes de secundaria. Adicciones, 13, 159-171.

Secades Villa R. y Fernández Hermida, J.R. (2001). Investigación sobre hábitos de consumo, factores de riesgo y de protección en jóvenes de Oviedo 2001. III Jornadas sobre Municipio y Drogodependencias. Oviedo, 26-27 de noviembre. 
